\title{
I-C-E Framework: Concepts for Group Dynamics Research in Human-Robot Interaction
}

\author{
Revisiting Theory from Social Psychology on Ingroup Identification (I), Cohesion \\ (C) and Entitativity (E)
}

\section{Anna M. H. Abrams • Astrid M. Rosenthal-von der Pütten .}

Received: date / Accepted: date

\begin{abstract}
The research community of human-robot interaction relies on theories and phenomena from the social sciences in order to study and validate robotic developments in interaction. These studies mainly concerned one (human) on one (robot) interactions in the past. The present paper shifts the attention to groups and group dynamics and reviews relevant concepts from the social sciences: in-group identification (I), cohesion (C) and entitativity (E). Ubiquitous robots will be part of larger social settings in the near future. A conceptual framework, the I-C-E framework, is proposed as a theoretical foundation for group (dynamics) research in HRI. Additionally, we present methods and possible measures for these relevant concepts and outline topics for future research.
\end{abstract}

Keywords human-robot interaction · HRI · group dynamics · ICE framework · ingroup identification . cohesion · entitativity

\section{Introduction}

The field of Human-Robot Interaction (HRI) has developed over the last 20 to 30 years [1]. Studies in this field often involve a human participant and the robot, as an interaction partner. Considering potential, ubiquitous deployments of robots and their integration in daily future life, the scenario of one human and one robot interacting with each other in isolation will be unrealistic in most of the deployment scenarios. Robots will

\section{A. M. H. Abrams}

Chair Individual and Technology, RWTH Aachen University E-mail: anna.abrams@humtec.rwth-aachen.de

A. M. Rosenthal-von der Pütten

Chair Individual and Technology, RWTH Aachen University

E-mail: arvdp@humtec.rwth-aachen.de be co-workers, servants, maybe even companions, and thus, will be integrated in a social network consisting of more than only one human interaction partner, and possibly more than only one robotic partner.

Research in the field of social psychology investigating human groups shows that group-level processes are fundamentally different from individual-level processes. One of the earliest research in this field was done by Kurt Lewin who is known as the founder of field theory in social sciences [2]. The theory builds upon the assumption that individual behaviour results from personal and environmental factors, and the interaction of both. A group influences an individual's behaviour due to the individual's interaction with other members in a social setting. This is what Lewin called "interactionism". Group phenomena can only be understood when a group as a whole is studied on the group-level and cannot be fully understood by observing individuals, thereby ignoring group influences or social settings [3]. An example of group processes that cannot be explained by individual preconditions is the phenomenon of groupthink which is described as an erroneous process of decision making due to social dynamics in groups. Janis Irvin analyzed failed decision making processes by expert groups, among others the Challenger disaster where security concerns somehow got lost in the decision process which finally led to the tragic decision the spacecraft was ready for flight. His explanation of this erroneous decision making is "a mode of thinking that people engage in when they are deeply involved in a cohesive in-group, when the members' strivings for unanimity override their motivation to realistically appraise alternative courses of action" (1982, as cited by [4]). In other words, groupthink appears in groups that are highly cohesive which leads group members to commit mistakes because agreement 
is set as the highest goal. The example of groupthink shows why research on a group-level is so important. In consideration of findings from group dynamics research and the likely event of robots playing a part in social settings in the near future, research on robots and groups is necessary. Still, groups that interact with robots or robot groups are seldom studied, although the need for a paradigm shift has been acknowledged $[5,6]$.

\subsection{HRI and Group Research}

Many different fields and subjects of study have emerged that are involved in group research with artificial agents. Most research has addressed technical challenges to enable robots to identify, keep track of and attend to multiple humans in interactions. It has been researched in online studies and in interaction studies how humans perceive and evaluate robot groups and whether humans tend to prefer robots that were marked as ingroup members. In laboratory settings and observational studies in the field, researchers explored how interaction in dyads deviate from interaction in groups involving robots and humans with the goal to derive relevant concepts that need further investigation such as emotional climate, social signal modelling, group norms and so forth. In addition, robots have been used to positively shape interactions between humans.

\subsubsection{Technical Solutions to Handle Multiple Users}

New fields of research in HRI and other disciplines such as computer vision emerged to find technical solutions to perception of and behaviour in multi-agent interactions. For instance, regarding computer vision, a robot has to identify multiple objects of interest [7], e.g., potential interaction partners, decide upon relevant objects [8], and keep track of these relevant objects/people [9]. Scholars from the field of social signals processing come into play when a robot takes on the challenge to recognize and interpret social behaviours shown by the identified interaction partners. Human communication is multi-modal (verbal and nonverbal). 60-65\% of communication is nonverbal [10]. Using diverse mechanisms and nonverbal cues, we are able to structure our conversations, regulate turn taking, establish conversational roles, and convey intentions and emotional states and so forth. Especially communication management (e.g., turn-taking, back-channeling) and relational communication [11] are heavily based on nonverbal messages and have been addressed in HRI dyads for years. Hence, research groups now shifted to work on realising attention management, turn-taking gaze behaviour and other social gaze behaviour for robots in multi-party interac- tions (e.g., [12-14]). Motion in human groups has to be interpreted in real time to anticipate future actions of human group members and synthesize the robots' own motion accordingly [15].

\subsubsection{How Humans Perceive Robot Groups}

Other research groups concentrate on humans perception of entitativity of robot groups or social effects in minimal group paradigms. In online studies featuring pictures or videos of single and groups of robots, Fraune and colleagues [16] examined when a quantity of robots is perceived as a group and found that number, type, similar colour and synchronized behaviour lead to higher groupness (entitativity) perceptions of the observed robots. Synchronicity in movement and similarity in appearance in a group of robots was found to be perceived more negatively, i.e. threatening [17]. Eyssel and colleagues further investigated the impact of social categorization of social robots on its perception. When a robot is presented as an in-group member (German participants evaluate robot developed in Germany in contrast to fabricated in Turkey) participants show an in-group bias evaluating the robot developed in Germany more favourably [18]. Moreover, the ingroup robot was identified to be psychologically closer, warmer and having more mind [18]. The social category of robots was also investigated in a study done by Häring and colleagues [19]. Their results support earlier findings by Eyssel and Kuchenbrandt: the in-group robot was more positively evaluated and anthropomorphized to a higher extent. Taijfels minimal group paradigm [20] also shows effect in HRI. Participants who were assigned into a blue group together with a robot showed an extent of anthropomorphic inferences about the robot and more positive evaluations compared to participants in the condition were the robot was not in the same group [21]. Similarly, intergroup bias can significantly affect how close humans are approaching an in-group robot and how much they trust the robots suggested answers with respect to task difficulty [22]. However, this research on perception of robot groups (online studies) and minimal group paradigms (one robot grouped with one human) has so far not been conducted in group settings that go beyond the dyad.

\subsubsection{Group Dynamics in Human-Robot Groups}

First studies have begun to examine group dynamics in interactions between humans and robots. The research predominantly looks into how group interactions differ from interactions in dyads. For instance, engagement or disengagement with the interaction is expressed differently according to the type of interactions and changes 
across the group size in HRI [23]. Hence, robots should have different prediction models and, depending on the number of people around it, use the most appropriate (p.104). Alves-Oliveira and colleagues developed a framework to distinguish individual-level and grouplevel emotional expression in interactions in HRI and introduced the concept of emotional climate to HRI [24]. Observational research of robots interacting with human individuals and groups in the wild show ample indicators of how the constellation of different groups encountered by a robot shapes interactions - often with the result that the robot is unequipped to handle the situation efficiently and socially adequate [25,5]. Moreover, researchers investigated in interaction studies the influence of the size of a robot group, for instance with regard to how well participants can detect those robots which indicate attention toward the human by gaze behaviour [26]. The research team of Malte Jung investigates how a robot might positively intervene in interactions between two or more humans to moderate working team conflicts [27] or conflicts between children competing for toys [28] or to shape conversational dynamics for equal consideration of all group members inputs in a discussion [29].

\subsection{What is a group?}

Considering the described studies from the field of HRI, some researchers have already begun to study groups involving humans and robots. In order to study phenomena that define groups, a definition of the term "group" itself is essential. A group is commonly described as an assembly of two or more individuals, where two individuals are referred to as a dyad and three as a triad. Larger groups, namely mobs, crowds and other collectives classify as groups as well [30]. A dyad is a very special form of group and will be discarded in the present paper for two main reasons:

- There is a considerable amount of doubt whether dyads are in fact groups. Dyads can be very intimate and unique groups and thus belong to their own category for research [31].

- Dyadic interactions have been primarily studied in the HRI community [5] and the present paper is supposed to shift the attention from only dyadic interactions to triadic and more.

Consequently, groups are defined as an assembly of three or more agents. In this definition, agents can be human or robotic.

Human groups are described and defined by concepts such as entitativity and cohesion. These concepts have been studied extensively in social psychology. In general, the field of HRI is in need of well-studied and validated sociological and psychological phenomena and concepts to work with. As Irfan and colleagues describe, many researchers in this field are engineers who begin with building and designing a robot. When it comes to testing the robot in a study, some researchers are confronted with their lack of training and experience in fundamental theories of the social sciences and empirical methodology [32]. Especially, for non-social scientists a common framework from the social sciences can be a valuable tool. However, for sociologists and psychologists working in the field, a common framework is equally necessary. This is not only the case because the replication crisis has put much doubt on the validity of research results in the social sciences [32] but because some fundamental theories on groups from the $1950 \mathrm{~s}$ or 1960s have become fuzzy over time. Many times, social psychologists have used entitativity and cohesion interchangeably or cohesion was used to define what entitativity is [33]. As was to be expected, this fuzziness has entered research on groups in the context of HRI, because researchers rely on social psychology research. Scholars in HRI have used the words 'cohesive' and 'unified entity' to give a definition for entitativity [34] and state entitativity is defined as cohesiveness [...]" [5]. In order to sharply distinguish both concepts, literature from the beginnings of the conceptualization of cohesion and entitativity is reviewed in the present paper. Furthermore, the concept of in-group identification is integrated in the model as it has been identified as a highly relevant concept for new groups [35]. As group of robots and multi-agent groups (human-robot groups) are still very uncommon in society, the concept of in-group identification will be reviewed here.

\subsection{Objectives}

The present paper provides a brief overview of three very relevant concepts in group research: entitativity, cohesion and in-group identification. Subsequently, a theoretical framework integrating all concepts based on theories from sociology and psychology is presented that gives an overview on their relation and differentiation. Social psychology is largely influenced by psychological and sociological theories. Roughly, these two areas can be differentiated by the focus and perspective of research: while psychologists are largely interested in intra- and interindividual phenomena, sociologists are concerned with a societal perspective. In group dynamics research, both perspectives together deliver a holistic understanding and are of equal importance. Influencing theories in the field of group dynamics stem from both areas. In the present paper and the presented 
framework, the two perspectives are considered through the different levels in which the three concepts entitativity, cohesion and in-group identification are located: the group-level and the individual-level.

However, understanding a theoretical concept does not necessarily mean one knows when and how to measure it. In the last section of this paper, we discuss from which perspectives the concepts can be assessed and which methods are adequate to measure the concept, because a clearly differentiated theoretical underpinning and well-planned operationalization of studied constructs are of great importance for the HRI community.

\section{Reviewing Entitativity: A Group-Level Factor}

Entitativity, meaning the perception of social groups, is seen as a fundamental antecedent of many phenomena studied in social psychology [36]. The term entitativity was coined by Campbell who proposed to apply empirical evidence from visual perception of physical entities to the perception of social groups [37]. Influenced by Gestalt principles such as proximity (elements that are close to each other are grouped together), similarity (elements that are alike are seen as belonging together), common fate (elements moving in the same direction form an entity) and good continuation (elements forming patterns are grouped together), Campbell described the perception of belongingness and their boundaries of social groups as entities. Entitativity is the degree of having the nature of an entity [37]. Still, 40 years later, the concept of entitativity seems to be based upon Campbell's proposal. In three studies, Lickel and colleagues examined factors that differentiate groups from one another and those factors' relation to the perception of the groups' entitativities [38]. They give the example of an ethnic group and a family that differ in terms of group size, the number of interactions among group members and many other factors (e.g. group size: ethnic group is large, family group is small). In their article, they attempt to find a common framework for studying groups and show that groups are perceived differently concerning their entitativity or "groupness". Variables such as perceived interaction (e.g. amount and intensity of interactions between group members), common goals (e.g. all members of a sports team want to win a game), common outcomes (e.g. a working team presents their solution for a problem), similarity of group members (e.g., similarity in appearance: same hair color or skin tone, same clothing style, wearing uniforms, speaking the same language) and importance of the group are strongly correlated with entitativity. Moreover, group size, duration (e.g. long-lasting group like family or incidental group such as a group of people waiting for the bus) and permeability (e.g. a family is hard to get into vs. you are easily a new member of the group waiting for the bus) are correlated to a weaker extent with perceived entitativity [38]. They tried to answer the question of when a conglomerate of individuals were actually seen as a group, an entity, and when they were simply seen as individuals. Conducting a cluster analysis on participants' perceptions of the Gestalt principles mentioned above, they identified four categories of entitativity: intimacy groups, task groups, loose associations and social categories. In their experiments they let participants indicate how they would rate different conglomerates of people from "not at all a group" to "very much a group". In total, 16 different conglomerates were rated using the VAS. Results showed, that loose associations such as a waiting line at a bank or people at bus stops were seen on the lower end of the concept of "groupness" while intimacy groups such as families and task groups with strong common goals such as sports groups were sorted to the upper end. Medium scale ratings were given to social categories such as "women" and other task groups such as "work teams".

Ip studied the influence of gestalt principles on perceived entitativity of groups [39]. In their studies they used cartoon aliens to exemplify different constellations. Different from the previously described study by Lickel et al. [38], here, the focus was on finding out which specific cues led participants to see a group as entitative [39]. Thus, participants were given different information on the cartoon aliens, e.g. a group of aliens that was physically similar or that moved synchronously. They found: similar ratings of entitativity resulted from different information. Furthermore, the authors describe the mediating role of perceived common traits for the perception of entitativity when physical similarity was the cue. In addition, the perception of common goals mediated the relationship between synchronous movement and the rating of entitativity [39]. A rather complex relational model of cues is the result of this research. The main finding, however, is that the extent of entitativity was rather independent of the cue given: the ratings were similarly high or low. These findings are consistent with a theory of the formation of perceived entitativity. The theory postulated that a group can be perceived as entitative resulting from two distinct ways: through a categorical construal and a dynamic construal. Depending upon the information an observer has, he or she base their perception upon either categories or dynamics. Both, perceived similarity 
of group members (a categorical construal) and perceived interaction (a dynamic construal) were found to be antecedents of entitativity [36]. The reported findings suggest that entitativity does not depend on a single cue or factor, but is rather based upon many cues independently, or a combination of them [33].

In general, the more diverse a group's members are perceived and the bigger the group is, the less entitative it is perceived [40]. Does this statement hold true in any case? Are diversity and size of a group negatively correlated to entitativity? Rutchick et al. [36] theorize that, on the one hand, an entitative group that is defined by its category is perceived as sharing common traits that are long-lasting. On the other hand, dynamic groups can be diverse and be perceived as entitative as long as they share agency characteristics, for instance, behavioural tendencies.

When reviewing entitativity research, it becomes apparent that entitativity is operationalized by subjective observer ratings and should in consequence be called "perceived entitativity". In consideration of the fundamental beginnings of conceptualizing entitativity, meaning its origin in Gestalt principles, the observer perspective seems inherent to the concept of entitativity. Furthermore, as an external observer only perceives the group as a whole, the description of groupness is based on the group level, rather than on an individual level. An observer is unable to infer how strongly related the group really is and how strongly related each individual feels towards other members of the group.

To conclude, the concept of entitativity defines the perceived groupness of a social unity from an outsider's perspective. For an assessment of entitativity, outside observers rate the unity of a group. The concept exists on the group-level only.

\section{Reviewing Cohesion: A Group-Level and Individual-Level Factor}

Cohesiveness was first described as the willingness to stick together in a group and stems from research by Kurt Lewin and colleagues [41]. Cohesion was later defined as a set of forces that keep members together: attraction and repulsion [41]. In contrast to entitativity, that describes how a collective is perceived as a unified entity, group cohesiveness is described as the actual degree to which a group is unified and coherent [38]. Additionally, cohesion has been named in the context of small groups and named the most important variable of small groups [42]. Still, there is a considerable amount of unclarity around the term. The above mentioned descriptions and definitions of cohesion do not indicate whether cohesion depends on factors on

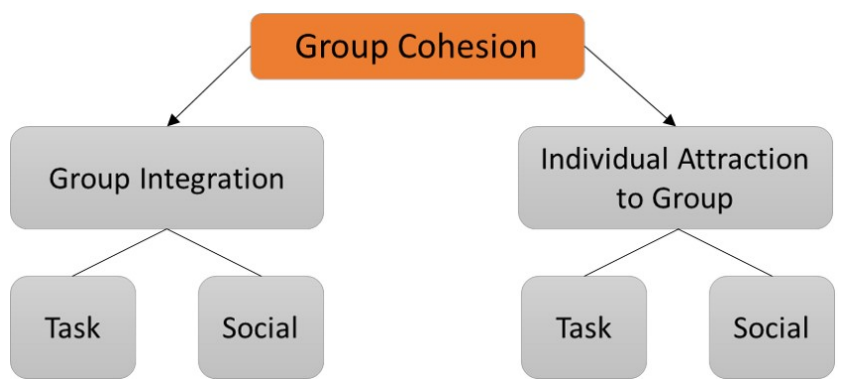

Fig. 1 Carron's hierarchical model of group research [45]

the individual level, such as a members attraction to the group, or on the group level, such as the presence of other groups [33].

In 1950, Festinger and his students Schachter and Back studied cohesion of two housing units at MIT [43]. They defined cohesion as "the total field of forces which act on members to remain in the group" (p. 37) and distinguished between attractiveness of the group and group goals. Dion describes this definition as the beginnings of the differentiation of social and task cohesion [41]. From their studies, Festinger and colleagues concluded that the higher the group's cohesion, the less deviating behavior was shown [43]. A review of the conceptualization of cohesion from field of forces to a multidimensional construct was done by Dion in 2000 [41]. Dion explains that Festinger and colleagues distinguished between the housing units and subgroups, e.g. closer friendships, and set standards for further group research [41]: there is not only one kind of group, groups have different qualities.

Many studies between the 1940s and 1970s involve factor analyses disentangling individual and group variables (e.g. [44]). With the use of factor analyses, researchers started to view cohesion as a multidimensional concept. An influential model stems from research conducted in the field of sports psychology where group processes play an important role. This model integrated the multidimensional view of cohesion [41]:

Carron [45] developed a model and an instrument to assess cohesion, the group environment questionnaire (GEQ). Carron and colleagues defined cohesion as a twofold construct: on the individual level and on the group level [46]. The individual level involves a members perception and evaluation, e.g. attraction towards the group (see Fig 1). The group level includes factors such as team members perceptions of similarity within the group and closeness to the whole group. In a factor analysis, the concept of cohesion with its two levels was further researched and found to consist of two more dimensions besides the individual and group differentiation. The following sub-scales are thus reflected in the GEQ [46]: 
- Group integration - task (GI-T): individual group member's perception about similarity and closeness with regard to the task at hand; e.g. Our team is united in trying to reach its goals for performance.

- Group integration - social (GI-S): individual group member's perceptions about group's similarity, closeness and bonding concerning its status as a social unit; e.g. Our team would like to spend time together in the off season.

- Individual attraction to group - task (ATG-T): individual group member's feelings about personal involvement in the group task, the groups goals and objectives; e.g. I'm not happy with the playing time I get (reverse scored).

- Individual attraction to group - social (ATG-S): individual group member's feelings about personal involvement and acceptance in the group's social activities; For me, this team is one of the most important social groups to which I belong.

In the present paper, this twofold level approach is adopted for all concepts and integrated in the below described conceptual framework: group-level and individual-level. Much of the research on cohesion was conducted with groups such as sports teams, therapy groups [33], and in the military [41]. Hence, research on cohesion normally involves well-established groups. This gives a hint on how cohesion is conceptualized in social psychology. In other words, cohesion is described as resulting from group development processes that lead to an "intense" group [3]. How does a group develop cohesion? The classic group development theory by Bruce Tuckman gives an explanation [47]. Group development comprises 5 different stages: Forming (the orientation stage which consists of self-disclosure and information gathering among the newly formed group's members), storming (a stage dominated by conflicts and a lack of unity), norming (called the structure stage where the group forms norms, roles and cohesion), performing (performance stage) and adjourning (dissolution stage). Especially, the third stage is of importance for cohesion formation according to Tuckman. Establishing norms and rules form a better unity and the group becomes a more intense experience for its members [3]. However, this unanimity of a group can lead to negative consequences such as groupthink [3]. As explained in the introduction part of this paper, groupthink occurs in groups that are highly cohesive and leads to decision-making processes that can have fatal outcomes (cf. Challenger disaster, [4]) On the contrary, cohesion can have positive effects. Cohesion was found to be positively correlated to performance in real groups (not laboratory groups), in correlational studies (not experimental studies) and in small groups rather than in large groups [48]. Does cohesion cause good performance? Forsyth and colleagues found out that groups that succeed in a task report to feel more cohesive after their performance [49]. After reviewing studies on the cohesion-performance relationship, Forsyth summarizes the relationship is bidirectional: "Cohesion makes groups more successful, but groups that succeed also become more cohesive" (p. 156, [3]).

To sum up, cohesion is a quality that develops in a group and that is measured by asking the group's members to rate the group's groupness on two different levels (individual and group), in two different categories (task and social). It has mostly been researched in the context of established groups (sports teams, therapy groups, work groups, military groups) and in relation to performance.

\section{Reviewing In-group Identification: An Individual-Level Factor}

In order to understand how humans start to identify with a group (cf. section 4.3) they are or become a member of, we first have to understand how humans categorize themselves into groups (cf. section 4.1) and why they do so (cf. section 4.2). In the following subsections, first of all, the theories of social identity and self-categorization are explained. Thereafter, in-group identification is described.

4.1 How humans categorize themselves into groups Social Identity Theory

Humans socially categorize themselves and others in order to create a sense of belongingness. Belongingness serves many functions, among others it gives people an idea how to view and treat others [50]. The social identity theory (SIT) by Tajfel and Turner from the late 1970s defines "that part of an individual's selfconcept which derives from his knowledge of his membership of a social group (or groups) together with the emotional significance attached to that membership" (Tajfel, 1974, p. 69 as cited by [51]. The theory addresses the borders of individuals thinking in terms of "I" and "We". Very little manipulation is needed for people to feel part of an in-group which is shown by Tajfel in his minimal group studies. Simply assigning participants to one group or the other leads them allocate more resources to their own group. Later termed the "mere categorization effect", simple verbal categorization of individuals into different groups is sufficient to make people think "we" instead of "I" and behave differently towards the own group and the other [51]. 
Ellemers and Haslam summarize three main issues addressed by the social identity theory [51]:

- social categorization: psychological processes that differentiate an individual's personal identity from the social identity

- identity management strategies: different strategies for deriving a positive social identity

- socio-structural characteristics: characteristics of the social setting that are influential for adaption of different strategies

The theory proposes the notion that the group can be found within the individual [52]. However, SIT is not only important to explain individuals' tendencies to categorize themselves and others, but to differentiate groups from one another [3]. Even though SIT provides many explanations for group phenomena, the theory does not give a full answer to the question why people categorize themselves.

4.2 Why humans categorize themselves into groups Self-Categorization Theory

The question of why people tended to categorize themselves on a group-level rather than on an individuallevel was answered by Abrams and Hogg in the late 1980s: distinguishing successfully between groups enhances self-esteem and a threatened self-esteem benefits from and motivates intergroup bias [53]. As an individuals self-esteem is dependent upon group membership, strategies to protect the group and differentiate it from other groups are important for the individual. In line with these findings, the definition of the self in terms of a group-level factor was proposed by self-categorization theory (SCT). SCT distinguishes between different levels of categorization (e. g. someone can self-categorize as German, as a woman, and as a psychologist) [51].

To sum up, SIT gives an explanation on social categorization of oneself and others which serves to mark off different groups and derive a positive social identity. SCT builds upon SIT and gives an insight in the reason why people engage in social categorization. In the present paper, both (social identity and self categorization) are understood as concepts on the group-level. Social identity and self categorization processes depend on differentiating groups $[53,51,3]$. In order to categorize oneself to one group, one has to create an overview over other groups and be able to distinguish them. This is understood as perspective taking on the group-level.

The beformentioned theories (SIT and SCT) are different from the concept of ingroup identification. An individual might readily self-categorize into a certain group, but that does not necessarily imply a strong identification with that group. Hence, the concept ingroup identification, especially, the process of how someone comes to identify with a certain group, becomes relevant.

\subsection{The Development of In-Group Identification}

A review on the concept of in-group identification was done by Leach and colleagues in 2008. They explain five components of in-group identification that will be reviewed very shortly (cf. [54] for a broader insight):

1. Individual self-stereotyping: the perception of oneself as a group member, the tendency of self-depersonalization and feeling similar to other members

2. In-group homogeneity: the perception of oneself as an in-group prototype

3. Satisfaction: positive feelings towards the in-group

4. Solidarity: the feeling of a personal bond and commitment to the in-group

5. Centrality: in reference to SCT, the perception of the group as a central aspect of an individual's self

Based on Durkheim's differentiation on mechanical and organic solidarity with groups [55], two factors, on a higher order and more abstract than the five components above, have been found to make up in-group identification: self-definition and self-investment [54].

- Self-definition is said to be the passive process of self-categorizing "or the mere inclusion of the self in an in-group" (p.428) and is found to be related to self-stereotyping and in-group homogeneity [56].

- Self-investment is considered to be an active choice for a group and leads to actual investment into the group and thereby related to the other three components (satisfaction, solidarity and centrality).

The multidimensionality of in-group identification has been tested with the identification to the group of humanity, "the highest level of social abstraction", (p.426, [56]). The Identification With All Humanity (IWAH) scale was developed to measure identification with the "human" in-group [57]. In three studies, Reese and colleagues found the proposed underlying structure of the two factors. However, mere self-definition as a human was not sufficient to provoke a behavioural outcome. Self-investment indeed proved to be the more active factor, that led people to act (here, donate money) [56].

A lot from SIT and SCT have influenced the conceptualization and understanding of in-group identification, as shown by Leach's review [54]. How is in-group 
identification differentiated? In this article, the differentiation is considered to depend upon on which level we locate the concept.

\subsection{Conceptual Location of In-Group Identification}

The question whether in-group identification is a variable on the group- or individual-level is a complex one. SIT brings the group into the individual [52]. Likewise, group-level variables such as the group's size, status and salience affect the degree of in-group identification. Current group dynamics researchers argue for a multilevel understanding of in-group identification [58]. In order to be able to differentiate between categorization processes (from SIT and SCT) and a persons individual identification with a group, in-group identification is understood as an individual-level variable in the context of the I-C-E framework outlined in this paper. Furthermore, recent findings give an insight in the nature and development of in-group identification and show us, why the HRI community (human-robot groups) should conceptualize in-group identification on an individuallevel, while social psychology and group dynamics researcher (human groups) shift their view to a multi-level concept.

First, an example: A brown haired person might not identify themselves as much as a brown haired person as a red haired identifies themselves with the group of red haired people. The group of red haired is probably much smaller, thus, more salient, and might even have another status. In Breda, the Netherlands, there is a festival called The Redhead Days where thousands of people with red hair meet annually. The fact that this group has a particular status might be the reason why an individual is prone to feel identified with the group. This would be considered a group-level influence. However, the simple fact that an individual has red hair makes the person eligible to belong to the group of red haired. This rather trivial example shows how group membership depends on individual prerequisites. So, the individual plays a decisive role to which group one belongs to or not. Individual traits were found to be powerful determinants of the occurrence of in-group identification [59]. Obviously, it can get much more complicated than hair colour. Group belonging becomes more and more complex, the more intra- and interpsychological processes play a role in group formation and in-group identification.

For instance, one group-level influence is the time span a group exists. The longer a group persists, the more the in-group identification will merge with grouplevel influences [58]. Considering the duration of existence, unknown and new groups (e.g., human-robot groups) are a special case. Groups that are unknown to the individual are unlikely to influence an individual's decision to join the group [35]. An individual does not know the group, the group's qualities and its individual members. Thus, initial identification processes rely on internal, personal representations of the group and the evaluation of the own individual fit. Through self-anchoring processes, the self is projected onto the group [35] [60]. This stands in utter contrast to SIT and SCT where the group is said to be within the individual. When a group's identity is unclear and undefined, the individual decides, consciously or subconsciously, to identify with one specific group because of an internal belief that group membership will be a good fit. This definition is based on the individual, not on the group.

In this framework proposed for the HRI research community, a clear conceptualization of in-group identification as a variable on the individual-level is reasonable, since human-robot groups are new and unknown groups to most people. Group dynamics research in psychology and sociology was focused on human interaction. With robots entering the field as possible coworkers, servants and companions, new dynamics will emerge [61]. Considering the findings reported above, robots, as a new group of social agents, and humanrobot groups, as unknown conglomerates, will not trigger defined mental representations. Thus, individuals are unlikely to feel identified with robot groups and human-robot groups, because they cannot draw on these mental representations, knowledge about status and so forth. Relevant concepts on a group-level, e.g. status, are not in an individual's mind when confronted with a group that does not have a defined social status yet. Research has shown that individuals use themselves and their personal identity as an anchor when confronted with new and unknown groups [58]. This is the reason why at this point in time, the community of HRI researchers should put an emphasis on the individuallevel of in-group identification. Once interaction increases, individual group members start using the group for self identifying processes [60]. In consequence, once humanrobot groups have become known social groups, a shift to a multilevel conceptualization (taking into account the recent findings of dimensions and components of in-group identification) will be needed.

In conclusion, in-group identification means projection oneself onto the group. It depends on individual prerequisites that make an individual eligible to belong to a group. Furthermore, as an individual-level variable, in-group identification plays a particular role when the group is still unknown to the individual. With passing time and development of a group, in-group identification cannot be considered an individual-level variable 
anymore. In the context of HRI, humans encounter unknown and new groups which leads us to consider ingroup identification on the individual-level.

\section{The I-C-E Framework for HRI Research: Ingroup Identification (I), Cohesion (C) and Entitativity (E)}

Drawing on our faceted review of in-group identification (I), cohesion (C), and entitativity (E), the purpose of our proposed I-C-E Framework as depicted in Figure 2 is to integrate the concepts and explain their relationship to each other.

We have carved out on which conceptual level the concept should be located - a group level or an individual level. Moreover, we specify whether the consideration of the concepts entail an inside member perspective or an outside observer perspective. In this regard, entitativity is conceptualized as a group-level variable from an outsider/ observer perspective. Cohesion can either be on the group-level or on an individual-level, but necessarily from an insider/ member perspective. In-group identification is conceptualized as an individual-level variable and can be measured through an insider/ member perspective. This encompasses methodological consequences regarding how the concepts can be measured (cf. section 6).

All three concepts are correlated. In the following paragraph, relations among the concepts are further explained.

\subsection{Entitativity-Cohesion Relation}

Prior research in Human-Human Interaction (HHI) as well as in HRI, entitativity and cohesion was often used interchangeably. Some researchers even wrote about the concept of "in-group entitativity" e.g. [52]). In-group entitativity would be an in-group member judgement about the groupness of the group. However, when considering entitativity and cohesion as reviewed above, it is clear that "in-group" entitativity would in fact be cohesion, because being part of a group, declassifies an individual to be able to be an outside observer. The outside observer perspective is a necessary requirement for the assessment of entitativity. The in-group member perspective automatically leads an in-group member to perceive the group's cohesion rather than its entitativity. Even though there is a strong correlation between a groups cohesion and its entitativity, clearly distinguishing these concepts will deliver a more distinct picture of the groupness of groups and sharpen the understanding of group processes.
However, many cue properties have been found to be predictors of both concepts, entitativity and cohesion [33]. For the differentiation of these concepts, these findings pose a challenge. Two studies on the relation of cohesion (task and social) and entitativity delivered high correlations between both concepts. While high correlations are often the case, there might be cases where assuming this correlation will lead to misjudgements. For instance, a family might be seen as highly entitative from an outsider perspective, but they may or may not regard themselves as cohesive from an insider perspective. An observer can neither perceive quarrels from the past nor observe a loss of feeling connected. A additional example can be given on a group of convicts standing in line for a bus: They might be perceived as a very entitative group based on their similar appearance in clothing and their shared fate. It is, however, very unlikely that the convicts, who just happen to be on the same bus, regard themselves as a cohesive group.

In order to unravel the concept of cohesion and entitativity, we have to take a closer look into their operationalization. Thurston conducted two studies on cohesion and entitativity and their relationship [33]. In both studies, Thurston examined the entitativity and cohesion of basketball teams. Entitativity was measured by a four-item entitativity scale, entitativity cues (such as interaction between members and shared goals) were assessed by nine items and cohesion was assessed though the 18-item Group Environment Scale (GEQ, cf. section $\mathrm{xx}$ ). In the first study, the questionnaires were given to the players directly after the game. This study delivered highly correlated results of entitativity and task cohesion, and correlations of entitativity and social cohesion to a lesser extent. Here, cohesion and entitativity was assessed by ingroup members. For them, it is impossible to assess how entitative the group is perceived from an observer perspective. In fact, their answers most likely reflected the same concept, namely participants' selfreported cohesion, on both questionnaires. This also means that high correlations are inevitable. In the other study, participants rated 18 basketball teams based on written description of the teams. Each description reflected one of the nine entitativity cues. The cues were taken from Lickel's eight entitativity cue properties [38], e.g. interaction between members, mutual group importance, shared outcomes and shared goals. One cue was added by Thurston: interpersonal liking among team members. Thurston explains why: it " was added due to the importance that interpersonal liking is typically ascribed in the cohesion literature" (p.51, [33]). These cue properties were positively correlated with entitativity, task cohesion and social cohesion [33]. 


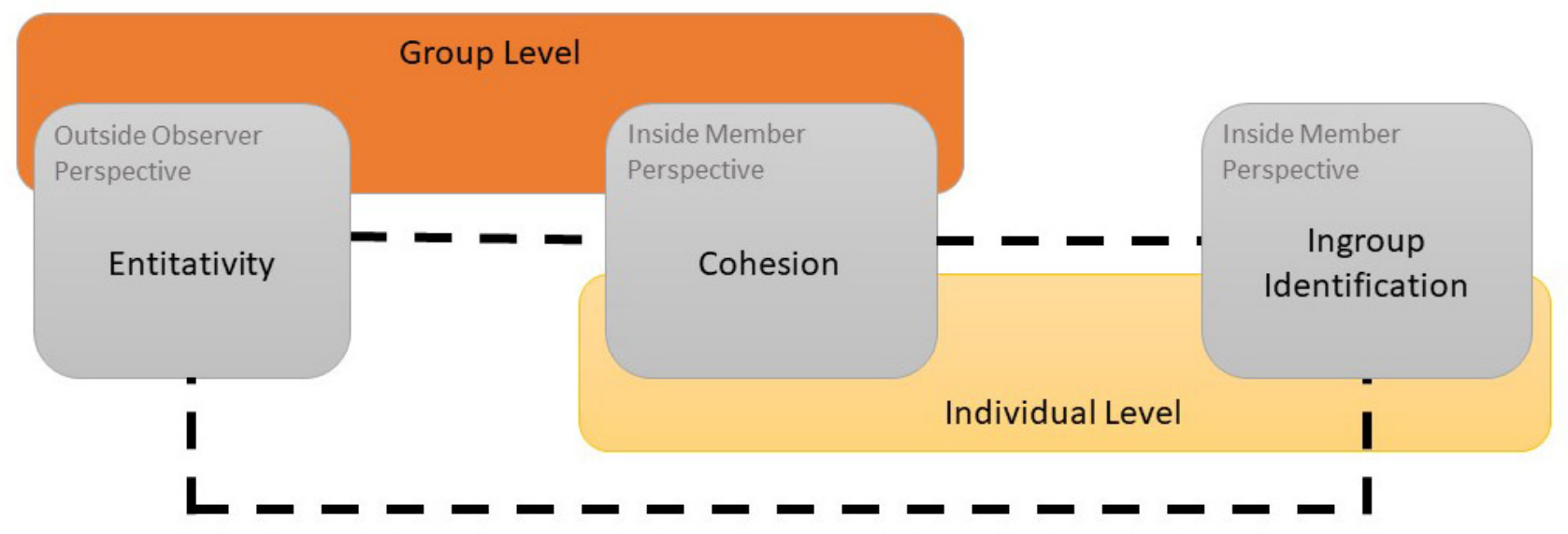

Fig. 2 I-C-E Framework for HRI Research

Reflecting upon these findings, one could argue that cohesion and entitativity might not be distinct concepts at all, or might belong to the same underlying factor. In light of the current I-C-E framework, the distinction between both concepts could not be found in the two studies because the perspective of measurement did not reflect the theoretical descriptions of entitativity and cohesion. Both concepts were measured using questionnaires given to a real team and later to a group of observers (lab study participants). High correlations among the concepts, given that many cue properties had been found to predict both concepts alike, are very logical. The same rater from the same perspective evaluated the teams' groupness: first, in-group members assessed their own in-group's entitativity and cohesion, second, observers assessed teams' entitativity and cohesion. In both studies, the same questionnaires for cohesion and entitativity were handed out. The internal validity of the assessment of both concepts is questionable. Was it really cohesion and entitativity, two distinct concepts, that were measured? The current framework proposes another understanding and approach: entitativity is a concept that can only be measured from an outsider or observer perspective and cohesion can only be measured from an insider or member perspective. The perspective is the main differentiation between entitativity and cohesion.

While entitativity, due to its measurement perspective (outside observer perspective), can only be measured on the group level, cohesion is more complex. On the one hand, cohesion, measured though evaluation of an in-group member, can be operationalized as an individual members attraction to the group, liking, importance, and other factors (inside member perspective, individual-level). On the other hand, cohesion can be an evaluation of the whole groups groupness made by an in-group member (inside member perspective, group-level). Building upon other research, decade after decade of research, concepts and their assessment had become fuzzy. Researchers studying the concept of cohesion studied solely cohesion and researchers interested in entitativity developed the concept of entitativity. Conceptualizations of social psychology constructs effect research in HRI as well.

In an exploratory study, group effects in interaction were studied with a robot in a public mall in Japan [5]. The robot actively approached humans once they were about one metre away from it and provided explanations for direction. After an interaction, people were approached by researchers and asked to fill in a survey on group size, group type, entitativity, ratings of the robot and some personal information. They found more entitative groups to be more positive toward the robot, to interact more and longer and to behave more socially [5]. However, the measurement of entitativity consisted of self-reports. Additionally, entitativity was defined as "i.e., cohesiveness with group" (p.5). In this study, cohesion rather than entitativity was assessed. The findings from this study should thus be reframed: More cohesive groups are more positive toward the robot, interact more and longer and behave more socially. However, the researchers could have considered to link cohesion with entitativity. By video taping the interactions, researchers could have shown them to participants in a lab and assess entitativity on Likert scales or by rating or sorting them, e.g. similar to Lickel et al. [38] or Ip et al. [39]. Self-reported cohesion and coded entitativity could be correlated.

Two years earlier, the same researchers conducted a study on the effect of entitativity on perceived threat and behavioral aggression towards robots [17]. This study used perceptional observer measurement methods for 
entitativity [17]. Entitativity was manipulated by a robot's appearance, motion, decision making and proximity. Participants assessed the robots' entitativity from an observer perspective with items such as "This group of robots should be thought of as a whole" (general entitativity), "These robots are affected by behaviors of other members of this group" (dynamic entitativity) and "These robots have similar physical appearances" (static entitativity). They found that entitative groups of robots were seen as more threatening in comparison to a single robot and a group of robots that had been varied in appearance only [17]. In this study, entitativity was assessed with observer's perceptions which corresponds well to the theoretical definition.

The relationship of entitativity and cohesion can be summarized as follows:

- Relation: Many cues (e.g. interaction between members, mutual group importance, shared outcomes and shared goals) have been found to predict cohesion and entitativity.

- Differentiation: Entitativity is a group-level variable and assessed through an outsider's perspective. Cohesion exists on the group-level and on the individual-level and is assessed through an insider's perspective.

When conducting research on either cohesion or entitativity of groups, the methods of gathering data play the most important role. Otherwise, internal validity of entitativity and cohesion is at stake. When reporting results of previous research, we would like to advise others to scrutinize measurement level and perspective in order to clearly distinguish between constructs.

\subsection{Cohesion-In-group Identification Relation}

When looking into the literature on in-group identification and cohesion, one finds out quickly that not only cohesion and entitativity get mistaken and used interchangeably, but the same problem exists for cohesion and in-group identification [62].

Henry and colleagues have proposed a model for ingroup identification and try to distinguish it from cohesion [62]. First of all, they explain how they relate: interpersonal attraction, relevant to cohesion, is a source of in-group identification. The developing bonds between group members heighten both, cohesion and in-group identification. The difference between both concepts is their relevance and meaning to the group and to the individual. While in-group identification is relevant to the individual, cohesion also exists on the group level [62].
In the field of cohesion research, researchers have put an emphasis on attraction (e.g. interpersonal attraction, attraction to the group task, attraction to the group's status). This notion has been criticized as it does not cover everything that binds individuals together to form a group [59]. Henry and colleagues argue that it covers an affective component of binding processes only. In-group identification also involves affective processes, but - in addition - cognitive and behavioural processes that lead an individual to identify with a group. Although in-group identification is conceptualized on an individual level, it can generate from different sources which can be on another level (e.g. selfcategorization processes or cohesion) [62]. Additional to various sources, the consequences of in-group identification effect both individual-level and group-level variables. Identification processes are essential for elicitation of group behaviours and enhance among others conformity, cohesion, intergroup competition, stereotyping and discrimination [63].

Another important differentiation can be made concerning duration. Duration is a cue predicting cohesion: the longer a group exists, the more cohesive it gets [3], [47]. In the beginning phase of group development, cohesion is nonexistent [47]. This is different with in-group identification. As known from research of new and unknown groups, an individual's in-group identification can be high even when joining the group was recent or the group is in the beginning phases of development. Van Veelen and colleagues have described this phenomenon by the self that is projected onto the group [35] [60]. To say it bluntly, for cohesion, the group (development) comes first, for in-group identification, the individual and its (conscious or subconscious) decision to identify with the group comes first.

In conclusion, the differentiation between in-group identification and cohesion is defined by:

- Time of Occurrence: In-group identification is already present when a group is encountered and cohesion develops within the group over time.

- Level of Existence: In-group identification happens within the individual (individual-level), but influences and is influenced by group-level characteristics such as cohesion (individual-level and group-level).

- Dependence on Individual Traits: While cohesion, on the individual level, is conceptualized to mainly depend on an individual's attraction to other members and the task, individual traits play a bigger role in initial in-group identification processes (the self is projected onto the group).

To our current knowledge, there are no studies on cohesion and in-group identification in multi-agent groups. 


\subsection{Entitativity-In-group Identification Relation}

The relationship between entitativity and in-group identification is embossed by very different understandings and operationalizations of entitativity. In a study on the relationship between entitativity, uncertainty and ingroup identification, entitativity was measured by one question: "How much of a group do you feel they are?". The question was asked to students on a campus who had previously indicated for which political party they voted. They had to think about other people who supported the same party and answer the question thinking about these other people [64]. It is questionable whether one question captures the concept of entitativity. However, the measurement problem lies within mixed perspectives. The student was asked to indicate how much they were a group. Because of the wording they, this measurement can be seen as a perceptual measure from an observer and might count as a measure for entitativity. Still, the student belonged to the same group which was made very salient before when he or she was asked for political orientation. How did they control that the student did not feel part of the group and considered him- or herself when rating the group's groupness (entitativity)? Afterwards, in-group identification was measured through nine questions adapted from social identity research [64]. Even though the title and hypotheses suggest a study on entitativity and in-group identification, a closer look into the methods gives aa different impression. What did they find? "Ingroup identification was influenced by the interaction of self-uncertainty [...] and perceived political in-group entitativity" (p.11, [64]; for a discussion of "in-group entitativity cf. also section 5.3). According to the current framework, these findings would have to be treated carefully.

Other studies on the relationship between entitativity and in-group identification rely on the same understanding of the entitativity concept and let in-group members rate their own in-group's unity (e.g. [65], [52], [66], [67]. Lickel and colleagues studied the perception of entitativity from an outsider perspective in two studies and afterwards perception of entitativity from an insider perspective [38]. Although he found the same 4 categories (intimacy groups, social categories, loose associations, task groups), cues such as importance of the group to the self, rather than group-member interaction, was most strongly related to entitativity in the ingroup condition. Duration was stronger positively correlated and permeability was stronger negatively correlated to entitativity. Also, group size was unrelated to entitativity in the in-group condition, but related in the outside perspective condition [38]. This provides ev- idence that entitativity from an outsider perspective is differently related to the predictive cues and that belongingness and/ or in-group identification does play a role in perception of a group. A clear cut between the perspective of research (insider/ member vs. outsider/ observer) as proposed in our framework is very relevant and necessary in order to control for confounding effects of, e.g. in-group identification, when researching entitativity.

The relationship between in-group identification and entitativity is embossed by an understanding of entitativity that is scrutinized in the present paper. The current understanding of the differentiation between entitativity and cohesion suggests that most of the discussed studies involve a perception of cohesion by an in-group member, rather than a perception of entitativity. Literature on the relationship between entitativity and in-group identification involves larger sized group than in research on cohesion and in-group identification. In a study by Castano and colleagues, participants had to rate their in-group's entitativity (Italian) with items such as 'Italians have many characteristics in common', 'Italians have a sense of common fate' and 'Italy has a real existence as a group' [52]. As in the above described study (political orientation), the group of Italians is a very large group. What becomes apparent is that researchers studying entitativity adapt a more societal perspective and examine rather large collectives instead of small groups. The confounding factor of group belongingness and/ or in-group identification might not be as highly influential in large collectives as in small groups but is most likely present. What does that mean?

When the relationship between entitativity and ingroup identification is under research and participants belong to the group under study it is very likely that they take themselves into account when rating the group's unity. An observer perspective cannot be occupied by an in-group member. Castano and colleagues found similar patterns of results for entitativity and in-group identification in their study and, in consequence, question the discriminant validity of these concepts. They even speculate that the distinction between entitativity and in-group identification might be artificial [52]. Conducting a factor analysis, they found two underlying factors which indicated that entitativity and in-group identification are not the same. Still, a clear overlap was found. Entitativity and in-group identification shared variance in further analyses of data [52]. We argue that this overlap is due to the perspective in measurement level. Entitativity is a group-level variable that should be measured from an outside perspective. In-group identification is conceptualized as an individual-level factor 
measured from an inside perspective (influencing and influenced by group-level factors in existing groups). In the discussed study, the individual-level was mixed into the group-level of entitativity: in-group members assessed entitativity (Italians assessed Italian unity). This explains very well why both concepts could not be clearly separated. Again, the importance of perspective and level of measurement is shown.

To conclude, reviews of the concepts of in-group identification, cohesion and entitativity show considerable overlap in the conceptualization, many cues predicting all three concepts and questionnaires with similar items assessing either one of them. From the 1950s and 1960s, where most research on group dynamics have its roots, the different concepts have spread and been researched by different researchers and branches in sociology and psychology. Small group researchers were mainly occupied with cohesion and researchers in the field of large groups concentrated on entitativity. In-group identification has been named social identity, group identity and many more. The current framework that puts an emphasis on perspective and level is supposed to serve as a common ground for research in HRI. It can and should be extended to integrate more concepts from groups dynamics research. The framework can provide a theoretical basis for the common understanding of these concepts and a common approach for research.

\section{Methods: Using the I-C-E Framework for HRI Research}

Our review has shown how important it is to i) know precisely the concept under consideration and ii) take account of the levels and perspectives when it comes to measuring group phenomena. As discussed in the previous chapter, study results suggest that there is considerable overlap of the concepts entitativity, cohesion, and in-group identification. However, as we demonstrated, most of this identified overlap can be ascribed to the lack of precise operationalization of the concepts. Hence, it is important to consider

- decide upon the level of measurement (individual vs. group)

- decide upon the perspective of measurement (insider/ member vs. outsider ( observer)

- decide upon the type of measurement (subjective vs. objective and qualitative vs. quantitative)

\subsection{How to Operationalize Concepts of I-C-E}

Consider the difference between individual- and grouplevel measurement: Group-level measurements involve questions about and assessments of the group as a whole, individual-level measurements involve questions addressing the individual.

Consider the difference between outsider/ observer and insider/member perspective measurement: An outsider or observer assessment requires a person that is not involved in the in-group, e.g. a participant in an experiment solely observing a group scenario (without being involved in the group). An insider or member perspective requires an in-group member to rate and evaluate the group.

Consider the difference between subjective and objective measurements: Subjective measurements concern an individual's perception or evaluation, e.g. an observer perceiving the groupness of a group or an ingroup member perceiving the groupness of a group; objective measurements involve structured methodology and do not involve evaluations

Consider the difference between qualitative and quantitative data: On both levels (individual and group level) and from both perspectives (outsider/ observer and insider/ member), qualitative and quantitative measurements can be used to extract data. Quantitative data result from structured observation and from questionnaires with, for instance, Likert scales. Qualitative data are gathered through interviewing and questionnaires that give option for free-text (e.g. "How do you perceive the group you observed? Write down what you think" for entitativity, "Explain in a few sentences how unified you think your group is." for cohesion and "Do you identify with the group and why? for in-group identification).

Hohman, Dahl and Grubbs state that "group entitativity is not an objective assessment of cohesiveness but is the perception of the degree of cohesiveness" [68]. In order to measure entitativity, one can ask an observer how cohesive a group appears (group-level variable). Asking an in-group member the exact same questions will not result in a measure of entitativity but an ingroup perception of the group's cohesion (group-level variable). The crucial difference between entitativity and cohesion lies in the perceptional perspective.

In Fig. 3, a group consisting of four agents is depicted that is observed by another agent. The picture shows a well-known social robot (pepper) and a fictitious robot arm. The picture visualizes a scene and will help to differentiate concepts and their level. In the following paragraph, the depicted scene is used to give examples of measurements: 


\section{The Group}

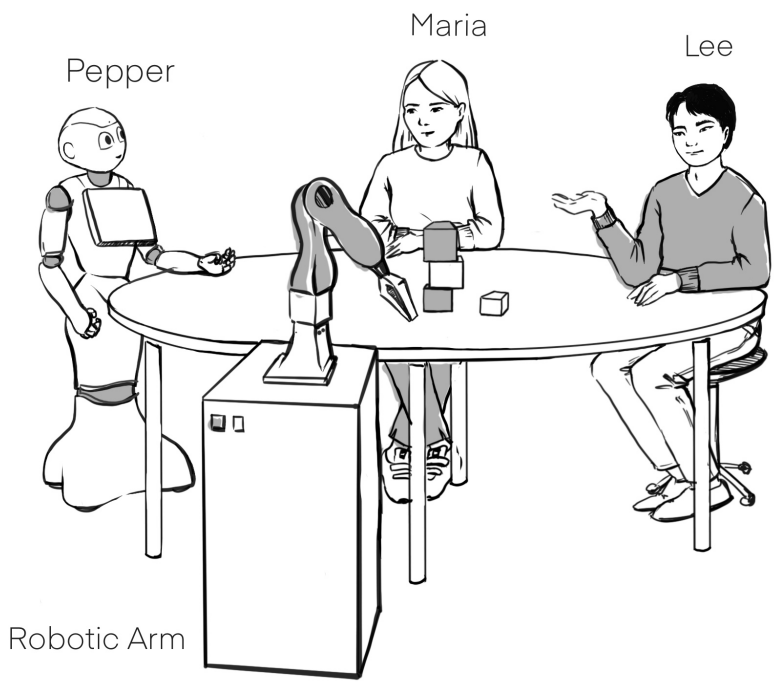

The Observer

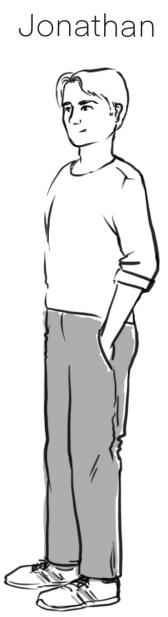

Fig. 3 Visualization: A group and its observer

\subsection{How to measure Entitativity - An Example}

Jonathan walks into a room and sees a group sitting at a table. If we ask Jonathan: "Do the agents around the table look like a group?", he sees two humans and two robots working together. He observes lively interaction and wooden blocks on the table that he infers to be involved in a common task. Group members interact and seem to have different roles. When Jonathan observes the scenario and rates the groups groupness, his rating reflects the group's entitativity. In this case, it is a very subjective perception. In a research setting, entitativity can be measured through independent raters observing interacting group members. For instance, just like Jonathan, raters would be asked to subjectively indicate how entitative the group appears to them. Interrater reliability can give an indication on the reliability and objectivity of these ratings. Lickel and colleagues have used a visual analogue scale to report ratings of groups. In order to rank groups according to their entitativity, the measurement is reasonable: it gives a relative measure of entitativity [38]. For absolute measures, is not advised to use. Other researchers have used scales with varying numbers of items rated on Likert scales in order to assess entitativity. As an example, the entitativity cue scale originally included eight items [38] and was extended by one item to also measure cohesion in one study [33]. Clear differences in scales measuring cohesion and entitativity are needed. Additionally, when a scale that is intended to measure entitativity is given to in-group members, the validity of measurement is at stake. Researchers would have to revise these scales in order to use them to correctly assess entitativity and carefully consider the measurement perspective (outside observer for entitativity).

6.3 How to measure Cohesion on a Group-Level - An Example

Maria sits at a table together with her work group. She has been working with this group for a week. If we ask Maria "Is your work group a real group?", she gives information on her groups status and talks about how well the group works together, that they 
have the common goal to teach a robot how to grasp objects. After some initial conflicts, she explains, the group has some shared outcomes: the robot arm exhibits some very precise grasping movements. When Maria describes the group progress and the groups dynamics, she describes its cohesion on a group-level. As a group member, Maria has insider information. She can talk about the groups real qualities. Comparing Marias and Lees perception of the group can give a broader insight in the groups cohesion on a group-level. Although self-reported data (Maria's and Lee's impression of the group's cohesion) seems the most reasonable for the assessment of cohesion in light of its theoretical definition, observational data can enrich the understanding of a group's cohesion. A very crucial differentiation has to be made in structured, observational measurements and an observer's perception (entitativity). Asking an observer for an evaluation measures entitativity. Observing a group and structuring the observations along well-defined criteria can measure cohesion. In order to conduct research with structured observation, well-validated approaches and well-trained researchers are necessary. A possible methodology to study cohesion on a group-level is the Interaction Process Analysis (IPA) by Bales [69]. The method has been used to study small groups and classify interaction into 12 categories belonging to either socio-emotional, relationship oriented interactions or to task oriented interactions. In light of current cohesion research (task and social), these two categories correspond very well and can be used as an observational measure. A subsequent development by Bales and colleagues is the Systematic Multiple Level Observation of Groups (SYMLOG) procedure which consists of 26 categories [70]. In order to create a rich understanding of a group's cohesion on a group-level, mixed-methods study designs (objective data, e.g. structured observation and subjective data through self-report; quantitative and qualitative data) are highly recommended.

6.4 How to measure Cohesion on an Individual Level An Example

Maria has been working with Pepper, the robotic arm and the newest group member Lee on a research project.

If we ask Maria: "Do you belong to the group?", she talks about how much she likes the interaction with the others in the group, especially Pepper, with which she has been working for a while. She feels very dedicated to the common task and has the feeling to be accepted. Here, Maria describes her group's cohesion on an individual-level. In contrast to the study of cohesion on a group-level, the study of cohesion on an individual-level can only be conducted by self-report measurements (e.g. questionnaires and interviews). A structured observation can only deliver results on a behavioral level. For an assessment of what Maria feels and thinks about their in-group, she has to be asked. Even though task and social cohesion are distinct from each other, they are correlated [33]. It is very likely that when Maria feels attracted to the group members (social cohesion), she will be more engaged with the group task (task cohesion). When Maria rates her interpersonal attraction high (individual-level cohesion), she will very likely rate the whole group's unity high (group-level cohesion). For an assessment of a working group's social and task cohesion the GEQ can be used as a measure for cohesion on a group-level and on the individual-level (see above, [45]).

\subsection{How to measure In-group Identification - An Example}

Lee has just joined the research project that Maria has been working on together with Pepper and the robotic arm. If we ask Lee: "How much do you think the group fits to you?, Lee might report the following. When his professor asked him which group he wanted to join, he explicitly named Maria's group. He is a very outgoing person and had seen Maria laugh and have fun while working together with Pepper and the robotic arm. Also, he thinks that the project is very innovative which matches his open-minded and creative character traits. The initial in-group identification with a multi-agent group can be of great interest to the community of HRI. What does a human lead to identify with such a group? As in-group identification is likely to evolve $[35,60]$, measuring the concept at the beginning of a developing group, during development and when established will deliver valuable insights. A scale to measure in-group identification on an individual-level was developed by Arrow and Carini, the Arrow-Carini Group Identification Scale 2.0 [62]. The scale reflects three different facets of in-group identification: affective (example item: "Members of this group like one another."), behavioral (example item: "This group accomplishes things that no single member could achieve.") and cognitive (example item: "I see myself as quite similar to other members of the group."). A downside of this questionnaire is its mere applicability to existing groups. For groups that have been experimentally sampled, e.g. minimal groups, this questionnaire is not well-fitted. Unfortunately, for recently encountered groups, the questionnaire cannot be used either. Inherent to the definition of in-group identification in 
the scale is an interaction component: in-group identification needs an actively interacting group [62]. In our understanding and concerning more recent research, ingroup interaction can be present before active interaction with a group [35], [60]. A scale for the initial and new in-group identification of humans (with a multiagent group) has to be developed.

\section{Directions and Questions for Future Work}

The field of social robotics and HRI relies on theories from the social sciences. The I-C-E framework provides a common ground for group (dynamics) research in the field, but we see this only as the beginning. Other concepts on group dynamics can be integrated by using the framework's differentiation of group- and individual level on the one hand, and the differentiation of inside-member or outside-observer perspective on the other hand. For instance, the work on intergroup bias is worth to be considered.

The previous chapter has demonstrated that although there are measures available to assess the concepts ingroup identification, cohesion, and entitativity, some of these measures should be revised in order to properly operationalize the concepts they shall cover. This is especially true for entitativity and cohesion.

With regard to methodology, we further face the task to create meaningful interaction paradigms in HRI to study characteristics of groups and their members. For this purpose, we have to identify which small group paradigms from social psychology research can be transferred and maybe adapted. Also, HRI specific scenarios have to be created, since by their nature, multi-agent groups are different from all-human groups. These differences, especially with regard to (social) capabilities of the robotic group members and hence agency perceptions of the robotic agents in comparison to the human agents are giving research on group dynamics a new twist. This latter overarching question finds its reflection on every concept level as the following potential future research questions will show:

Can a multi-agent group (human-robot group) be entitative? We would postulate that this is the case, as long as predictive cues such as similarity and common goals are somehow apparent to the observer. In accordance with Tennent et al. [29], who built a robotic device, MicBot, that shapes human group interaction, but was not designed to look human-like, future research could focus on the mechanisms that influence observers to perceive entitativity. A robotic team member, like the robotic arm in the drawing, might be used to find the outer boarders of entitativity. Would a robotic arm and MicBot be perceived as team members? Or is a robot only perceived as a member if it mimicks the human form and/ or human social behavior?

Can a multi-agent groups be cohesive? We would hypothesize that, given group members are attracted to the group itself (social cohesion) and committed to the common task (task cohesion), a multi-agent group can be cohesive. The specific circumstances and conditions are to be researched. Fraune and colleagues have postulated that the cohesion (cohesion according to this framework, although the authors refer to entitativity) can influence the quality of interaction of a human group with a robot [5].

Can humans identify with robot groups and multi-agent groups? The question of in-group identification with a group of robots might be the most interesting, as it is not yet understood and researched what might lead humans to identify with a robot (group). In contrast to entitativity and cohesion where some research in the field of HRI has been conducted and predictive cues give plausible hypotheses that multi-agent groups can be entitative and cohesive, in-group identification with robots is still a very open question. The simple prerequisite of red hair as an in-group identification factor, as in the example above, is not applicable to robots. Being a very novel constellation, a multi-agent group might generally provide the opportunity for more insights in the psychological processes at hand when groups and social identities are formed. As postulated by Reese and colleagues and their research on Identification With All Humanity [56], will the category of humanity stay the highest order of social abstraction? Or will there emerge an even more abstract level of intentional agents with the sub categories humans and robots?

As fascinating as human-robot interaction has been all these years, we look ahead to new and possibly even more interesting times when HRI scholars face countless new research paradigms on (social) group dynamics to be discovered as they boldly go where (almost) no one has gone before.

Acknowledgements The authors would like to thank Laura Platte (Chair Individual and Technology, RWTH Aachen University) for her drawing.

\section{References}

1. A. Weiss, in Proceedings of the seventh annual ACMIEEE international conference on Human-Robot Interaction, ed. by $\mathrm{H}$. Yanco (ACM, New York, NY, 2012), ACM Digital Library, p. 271. DOI $10.1145 / 2157689.2157789$

2. M.H. Kuhn, The ANNALS of the American Academy of Political and Social Science 276(1), 146 (1951). DOI $10.1177 / 000271625127600135$ 
3. D.R. Forsyth, Group dynamics, 6th edn. (Wadsworth Cengage Learning, Belmont, Calif., 2014)

4. I. Janis, IEEE Engineering Management Review 36(1), 36 (2008). DOI 10.1109/EMR.2008.4490137

5. M.R. Fraune, S. Sabanović, T. Kanda, Frontiers in Robotics and AI 6, 3 (2019). DOI 10.3389/frobt.2019.00048

6. M. Jung, P. Hinds, ACM Transactions on Human-Robot Interaction 7(1), 1 (2018). DOI 10.1145/3208975

7. M.D. Breitenstein, F. Reichlin, B. Leibe, E. KollerMeier, L. van Gool, IEEE transactions on pattern analysis and machine intelligence 33(9), 1820 (2011). DOI 10.1109/TPAMI.2010.232

8. A. Osep, W. Mehner, P. Voigtlaender, B. Leibe, in 2018 IEEE International Conference on Robotics and Automation (ICRA), ed. by K. Lynch, I.I.C.o.R.a. Automation (IEEE, [Piscataway, NJ], 2018), pp. 3494-3501. DOI 10.1109/ICRA.2018.8460975

9. D. Mitzel, B. Leibe, in Computer Vision - ECCV 2012, Lecture Notes in Computer Science, vol. 7576, ed. by D. Hutchison, T. Kanade, J. Kittler, J.M. Kleinberg, F. Mattern, J.C. Mitchell, M. Naor, O. Nierstrasz, C. Pandu Rangan, B. Steffen, M. Sudan, D. Terzopoulos, D. Tygar, M.Y. Vardi, G. Weikum, A. Fitzgibbon, S. Lazebnik, P. Perona, Y. Sato, C. Schmid (Springer Berlin Heidelberg, Berlin, Heidelberg, 2012), pp. 566579. DOI 10.1007/978-3-642-33715-4_41

10. J.K. BURGOON, A.E. Bacue, Handbook of communication and social interaction skills pp. 179-219 (2003)

11. J.K. BURGOON, D.B. BULLER, J.L. HALE, M.A. TURCK, Human Communication Research 10(3), 351 (1984). DOI 10.1111/j.1468-2958.1984.tb00023.x

12. M. Bennewitz, F. Faber, D. Joho, M. Schreiber, S. Behnke, in 5th IEEE-RAS International Conference on Humanoid Robots, 2005 (IEEE Operations Center, Piscataway, NJ, 2005), pp. 418-423. DOI 10.1109/ICHR.2005.1573603

13. A. Zaraki, D. Mazzei, M. Giuliani, D. de Rossi, IEEE Transactions on Human-Machine Systems 44(2), 157 (2014). DOI 10.1109/THMS.2014.2303083

14. V. Richter, B. Carlmeyer, F. Lier, S. Meyer zu Borgsen, D. Schlangen, F. Kummert, S. Wachsmuth, B. Wrede, in HAI'16, ed. by W.Y. Yau, T. Omori, G. Metta, H. Osawa, S. Zhao (The Association for Computing Machinery, New York, New York, 2016), pp. 43-50. DOI 10.1145/2974804.2974823

15. T. Iqbal, S. Rack, L.D. Riek, IEEE Transactions on Robotics 32(4), 909 (2016). DOI 10.1109/TRO.2016.2570240

16. M.R. Fraune, S. Sherrin, S. Sabanović, E.R. Smith, in HRI'15, ed. by J.A. Adams, W. Smart, B. Mutlu, L. Takayama (ACM, Associaton for Computing Machinery, [New York], 2015), pp. 109-116. DOI $10.1145 / 2696454.2696483$

17. M.R. Fraune, Y. Nishiwaki, S. Sabanović, E.R. Smith, M. Okada, in HRI'17, ed. by HRI (IEEE, Piscataway, NJ, 2017), pp. 205-213. DOI 10.1145/2909824.3020248

18. F. Eyssel, D. Kuchenbrandt, The British journal of social psychology 51(4), 724 (2012). DOI 10.1111/j.20448309.2011.02082.x

19. M. Häring, D. Kuchenbrandt, E. André, in HRI '14, ed. by G. Sagerer, M. Imai, T. Belpaeme, A. Thomaz (ACM, New York, 2014), pp. 9-16. DOI $10.1145 / 2559636.2559673$

20. H. Tajfel, M.G. Billig, R.P. Bundy, C. Flament, European Journal of Social Psychology 1(2), 149 (1971). DOI 10.1002 /ejsp. 2420010202
21. D. Kuchenbrandt, F. Eyssel, S. Bobinger, M. Neufeld, in Social Robotics, Lecture Notes in Computer Science, vol. 7072, ed. by B. Mutlu, C. Bartneck, J. Ham, V. Evers, T. Kanda (Springer Berlin Heidelberg, Berlin, Heidelberg, 2011), pp. 104-113. DOI 10.1007/978-3-642-25504$5 \_11$

22. C. Deligianis, C.J. Stanton, C. McGarty, C.J. Stevens, Journal of Human-Robot Interaction 6(3), 4 (2017). DOI 10.5898/JHRI.6.3.Deligianis

23. I. Leite, M. McCoy, D. Ullman, N. Salomons, B. Scassellati, in HRI'15, ed. by J.A. Adams, W. Smart, B. Mutlu, L. Takayama (ACM, Associaton for Computing Machinery, [New York], 2015), pp. 99-105. DOI 10.1145/2696454.2696466

24. P. Alves-Oliveira, P. Sequeira, F.S. Melo, G. Castellano, A. Paiva, ACM Transactions on Human-Robot Interaction 8(1), 1 (2019). DOI 10.1145/3300188

25. S. Sabanovic, M.P. Michalowski, R. Simmons, in The 9th IEEE International Workshop on Advanced Motion Control (IEEE, Piscataway, N.J., 2006), pp. 596-601. DOI 10.1109/AMC.2006.1631758

26. H. Admoni, B. Hayes, D. Feil-Seifer, D. Ullman, B. Scassellati, in 2013 8th ACM/IEEE International Conference on Human-Robot Interaction, ed. by I. Staff (IEEE, [Place of publication not identified], 2013), pp. 389-395. DOI 10.1109/HRI.2013.6483614

27. N. Martelaro, M. Jung, P. Hinds, in Proceedings of the Tenth Annual ACMIEEE International Conference on Human-Robot Interaction Extended Abstracts, ed. by J.A. Adams (ACM, New York, NY, 2015), p. 271. DOI 10.1145/2701973.2702094

28. S. Shen, P. Slovak, M.F. Jung, in HRI'18, ed. by T. Kanda, S. abanović, G. Hoffman, A. Tapus (Association for Computing Machinery, New York, New York, 2018), pp. 69-77. DOI 10.1145/3171221.3171248

29. H. Tennent, S. Shen, M. Jung, in 201914 th ACM/IEEE International Conference on Human-Robot Interaction (HRI) (IEEE, 3/11/2019 - 3/14/2019), pp. 133-142. DOI 10.1109/HRI.2019.8673013

30. G. Simmel, American Journal of Sociology 1902(8(2)), $158(1902)$

31. J.M. Levine, R.L. Moreland, A. W. Kruglanski \& W. Stroebe (Eds.), Handbook of the history of social psychology 2012, 383 (2012)

32. B. Irfan, J. Kennedy, S. Lemaignan, F. Papadopoulos, E. Senft, T. Belpaeme, in HRI'18, ed. by T. Kanda, S. abanović, G. Hoffman, A. Tapus (Association for Computing Machinery, New York, New York, 2018), pp. 1320. DOI $10.1145 / 3173386.3173389$

33. J.A. Thurston, (2012)

34. D.A. Effron, H. Kakkar, E.D. Knowles, Journal of Experimental Social Psychology 79, 239 (2018). DOI 10.1016/j.jesp.2018.08.002

35. R. van Veelen, S. Otten, N. Hansen, The British journal of social psychology 52(3), 543 (2013). DOI 10.1111/j.2044-8309.2012.02110.x

36. A.M. Rutchick, D.L. Hamilton, J.D. Sack, European Journal of Social Psychology 38(6), 905 (2008). DOI 10.1002/ejsp.555

37. D.T. Campbell, Behavioral Science 3(1), 14 (1958). DOI $10.1002 /$ bs.3830030103

38. B. Lickel, D.L. Hamilton, G. Wieczorkowska, A. Lewis, S.J. Sherman, A.N. Uhles, Journal of personality and social psychology 78(2), 223 (2000). DOI 10.1037/00223514.78.2.223

39. G.W.m. Ip, C.y. Chiu, C. Wan, Journal of personality and social psychology 90(3), 368 (2006). DOI 10.1037/00223514.90.3.368 
40. C. McGarty, S.A. Haslam, K.J. Hutchinson, D.M. Grace, British Journal of Social Psychology 34(3), 237 (1995). DOI 10.1111/j.2044-8309.1995.tb01061.x

41. K.L. Dion, Group Dynamics: Theory, Research, and Practice 4(1), 7 (2000). DOI 10.1037/1089-2699.4.1.7

42. K.A. Bollen, R.H. Hoyle, Social Forces 69(2), 479 (1990). DOI $10.1093 / \mathrm{sf} / 69.2 .479$

43. L. Festinger, Psychological Review 57(5), 271 (1950). DOI 10.1037/h0056932

44. R.B. Cattell, D.R. Saunders, G.F. Stice, Human Relations 6(4), 331 (1953). DOI $10.1177 / 001872675300600403$

45. A.V. Carron, W.N. Widmeyer, L.R. Brawley, Journal of Sport Psychology 7(3), 244 (1985). DOI 10.1123 /jsp.7.3.244

46. A.V. Carron, L.R. Brawley, Small Group Research 31(1), 89 (2000). DOI 10.1177/104649640003100105

47. B.W. Tuckman, M.A.C. Jensen, Group \& Organization Studies 2(4), 419 (1977). DOI 10.1177/105960117700200404

48. B. Mullen, C. Copper, Psychological Bulletin (115(2)), 210 (1994)

49. D.R. Forsyth, L.E. Zyzniewski, C.A. Giammanco, Personality and Social Psychology Bulletin 28(1), 54 (2002). DOI $10.1177 / 0146167202281005$

50. D. Abrams, M.A. Hogg, S. Hinkle, S. Otten, in Theories of small groups, ed. by M.S. Poole, A.B. Hollingshead (SAGE, London, 2005), pp. 99-138. DOI 10.4135/9781483328935.n4

51. N. Ellemers, S.A. Haslam, in Handbook of Theories of Social Psychology, ed. by P. van Lange, A. Kruglanski, E. Higgins (SAGE Publications Ltd, 1 Oliver's Yard, 55 City Road, London EC1Y 1SP United Kingdom, 2012), pp. 379-398. DOI 10.4135/9781446249222.n45

52. E. Castano, V. Yzerbyt, M.P. Paladino, S. Sacchi, Personality and Social Psychology Bulletin 28(2), 135 (2002). DOI 10.1177/0146167202282001

53. D. Abrams, M.A. Hogg, European Journal of Social Psychology 18(4), 317 (1988). DOI 10.1002/ejsp.2420180403

54. C.W. Leach, M. van Zomeren, S. Zebel, M.L.W. Vliek, S.F. Pennekamp, B. Doosje, J.W. Ouwerkerk, R. Spears, Journal of personality and social psychology 95(1), 144 (2008). DOI 10.1037/0022-3514.95.1.144

55. E. Durkheim, (G.Simpson, Trans.) New York:Free Press. (Original work published 1893) (1947)

56. G. Reese, J. Proch, C. Finn, European Journal of Social Psychology 45(4), 426 (2015). DOI 10.1002/ejsp.2102

57. S. McFarland, M. Webb, D. Brown, Journal of personality and social psychology 103(5), 830 (2012). DOI $10.1037 / \mathrm{a} 0028724$

58. L. Jans, C.W. Leach, R.L. Garcia, T. Postmes, Group Processes \& Intergroup Relations 18(2), 190 (2015). DOI $10.1177 / 1368430214540757$

59. K.S. Bouas, H. Arrow, Computer Supported Cooperative Work (CSCW) 4(2-3), 153 (1995). DOI 10.1007/BF00749745

60. R. van Veelen, S. Otten, N. Hansen, Group Processes \& Intergroup Relations 16(6), 671 (2013). DOI $10.1177 / 1368430212473167$

61. M.F. Jung, S. Šabanović, F. Eyssel, M. Fraune, in Companion of the 2017 ACM Conference on Computer Supported Cooperative Work and Social Computing, ed. by C.P. Lee (ACM, New York, NY, 2017), pp. 401-407. DOI 10.1145/3022198.3022659

62. K.B. Henry, H. Arrow, B. Carini, Small Group Research 30(5), 558 (1999). DOI 10.1177/104649649903000504
63. M.A. Hogg, D. Abrams, Florence, KY, US:Taylor \&Frances/Routledge. (1988)

64. M.A. Hogg, D.K. Sherman, J. Dierselhuis, A.T. Maitner, G. Moffitt, Journal of Experimental Social Psychology 43(1), 135 (2007). DOI 10.1016/j.jesp.2005.12.008

65. L. Gaertner, J. Schopler, European Journal of Social Psychology 28(6), 963 (1998). DOI 10.1002/(SICI)10990992(1998110)28:6<963::AID-EJSP905>3.0.CO;2-S

66. E. Castano, V. Yzerbyt, D. Bourguignon, European Journal of Social Psychology 33(6), 735 (2003). DOI 10.1002/ejsp. 175

67. V. Yzerbyt, E. Castano, J.P. Leyens, M.P. Paladino, European Review of Social Psychology 11(1), 257 (2000). DOI 10.1080/14792772043000059

68. Z.P. Hohman, E. Dahl, S. Grubbs, Self and Identity 15(6), 638 (2016). DOI 10.1080/15298868.2016.1185462

69. R.F. Bales, Interaction process analysis; a method for the study of small groups (Oxford, England: AddisonWesley., 1950)

70. R.F. Bales, S.P. Cohen, S.A. Williamson, Free Press (1979) 